\title{
Curcumin Reduces Tumour Necrosis Factor-Enhanced Annexin V-Positive Microparticle Release in Human Vascular Endothelial Cells
}

\author{
Antony Kam ${ }^{1,2}$, Kong M. Li ${ }^{3}$, Valentina Razmovski-Naumovski ${ }^{1,4,5}$, Srinivas Nammi ${ }^{4,5}$, Kelvin Chan ${ }^{1,4,5}$, Georges E. \\ $\mathrm{Grau}^{6}$, George Q. Li ${ }^{1}$ \\ ${ }^{1}$ Faculty of Pharmacy, University of Sydney, Australia; ${ }^{2}$ School of Biological Sciences, Nanyang Technological \\ University, Singapore; ${ }^{3}$ Discipline of Pharmacology, Sydney Medical School, University of Sydney, Australia; ${ }^{4}$ The \\ National Institute of Complementary Medicine (NICM), University of Western Sydney, Australia; ${ }^{5}$ School of Science \\ and Health, University of Western Sydney, Australia; ${ }^{6}$ Vascular Immunology Unit, Sydney Medical School and Bosch \\ Institute, The University of Sydney, Australia.
}

Received, May 6, 2015; Revised, August 17, 2015; Accepted, September 7, 2015; Published, September 9, 2015.

\begin{abstract}
PURPOSE: Circulating microparticles have been highlighted as biomarkers of cardiovascular disease state and progression. The aim of this study was to evaluate the effects of curcumin on microparticle release from endothelial cells undergoing TNF-induced cell activation and apoptosis. METHODS: This study evaluated the effects of curcumin on microparticle release, cytotoxicity, apoptosis, cell adhesion molecule expression and monocyte adhesion in EAhy926 human endothelial cells. RESULTS: The results showed that the numbers of microparticles were increased by tumour necrosis factor (TNF) or the combination of TNF and cycloheximide (CHX). Curcumin attenuated microparticle release caused by TNF or TNF plus CHX treatments. The pretreatment by curcumin not only negated the accelerated cell death and apoptosis caused by TNF and CHX, but also diminished TNF-induced cell activation, as assessed by reduced surface expression of intercellular adhesion molecule 1, and adhesion of monocytes to endothelial monolayers. CONCLUSION: Curcumin reduced microparticle release from endothelial cells undergoing cell activation and apoptosis, which supports its protective role in TNF-associated endothelial dysfunction, and highlights its potential use as a nutraceutical agent for vascular inflammatory diseases.
\end{abstract}

This article is open to POST-PUBLICATION REVIEW. Registered readers (see "For Readers") may comment by clicking on ABSTRACT on the issue's contents page.

\section{INTRODUCTION}

Turmeric (Curcuma longa Linn.) has been used extensively as a spice and herbal medicine for thousands of years. Medicine for many years. Curcumin (diferuloylmethane) is a yellowish polyphenolic component derived from the rhizome of turmeric. A large body of evidence has supported the anti-inflammatory effects and anti-cancer properties of curcumin (1-3). There is also accumulating evidence for its beneficial effects in cardiovascular diseases (1-4). In particular, it has been shown to block the production and effects of tumour necrosis factor (TNF) (5).

TNF (previously known as TNF- $\alpha$ ) is a pleiotropic pro-inflammatory cytokine implicated in cardiovascular disease and endothelial dysfunction (6). TNF induces endothelial cell activation through the upregulation of cell adhesion molecule expression and promoting monocyte-endothelium interactions (7). On the other hand, TNF can exacerbate apoptosis and cytotoxicity in the presence of transcription (e.g. actinomycin D) and translation (e.g. cycloheximide (CHX)) inhibitors $(8,9)$. Cell activation and apoptosis are the two cellular processes that can increase the shedding of cell-derived microparticles. Several reports have demonstrated that TNF strongly enhances microparticle formation (10-13). Cell-derived microparticles, also known as "cell dust", are small vesicles shed from the plasma membrane of activated or apoptotic cells $(14,15)$. Circulating microparticles originate from various cell types, including platelets, monocytes, endothelial cells, lymphocytes, erythrocytes and granulocytes $(16,17)$. Microparticles play an important role in cell-to-cell communications by transferring biological signals from host cells to the neighbouring cells, and thus having a substantial role on cellular homeostasis of the targeted cells (18). Elevated levels of circulating microparticles have been highlighted as independent predictors for

Corresponding Author: Dr George Q. Li, Faculty of Pharmacy, University of Sydney, NSW 2006, Australia; Email: george.li@sydney.edu.au 
cardiovascular disease events (19).

Although scientific evidence supports the cardioprotective effects of curcumin, the effect of curcumin on microparticle formation has not yet been investigated. Hence, the aim of this study was to evaluate the effects of curcumin on microparticle release from endothelial cells undergoing TNF-induced cell activation and apoptosis.

\section{MATERIALS AND METHODS}

\section{Materials}

Human TNF recombinant protein was purchased from Cell Signaling Technology (USA). Curcumin ( $>$ 98\% HPLC) was purchased from Chengdu Biopurify Phytochemicals Ltd. (China). Cycloheximide was purchased from Santa Cruz Biotechnology (USA). CellTiter ${ }^{\circledR}$ Glo Luminescent Cell Viability Assay Kit was purchased from Promega (Australia). JC-1 was purchased from Biotium (USA). R-Phycoerythrin (PE)-conjugated antibodies (CD54) were purchased from e-Bioscience (USA). Fluorescein isothiocyanate (FITC) Annexin V apoptosis detection kit was purchased from BD Pharmingen (Australia). Cell culture reagents were from Life Technology (Australia), and other chemicals were from Sigma-Aldrich (Australia) unless otherwise stated.

\section{Cell line and culture conditions}

Human cardiovascular endothelial cell line (EAhy926) was kindly provided by Dr Shanhong Ling (Monash University Central Clinical School, Australia). The cell line was cultured in DMEM/Ham's F12 containing $15 \mathrm{mM}$ HEPES and L-glutamine, supplemented with $10 \%$ foetal bovine serum and $100 \mathrm{U} / \mathrm{mL}$ of penicillin and streptomycin (Gibco BRL, Australia) (20). Human acute monocytic leukaemia cells (THP-1) were kindly provided by Dr Prasad Kota (University of Sydney, Australia) and were cultured in RPMI-1640 medium containing L-glutamine supplemented with $10 \%$ foetal bovine serum and $100 \mathrm{U} / \mathrm{mL}$ of penicillin and streptomycin (Gibco BRL, Australia). Both cell lines were grown in a $5 \% \mathrm{CO}_{2}$-humidified incubator at $37^{\circ} \mathrm{C}$.

\section{Cell viability assays}

Cell viability measurement by MTT dye reduction assay was performed as previously described (20). Briefly, EAhy926 cells were exposed to TNF \pm cycloheximide (CHX) for 24 hours after pretreatment with curcumin (final DMSO concentration $0.1 \%$ ) for one hour. MTT (final concentration $0.5 \mathrm{mg} / \mathrm{mL}$ ) solution was added and incubated for 4 hours at $37^{\circ} \mathrm{C}$. Dimethyl sulfoxide was then added to dissolve the insoluble formazan crystal. The absorbance was measured at $550 \mathrm{~nm}$.

Cell viability was also assessed using the CellTiter $^{\circledR}$-Glo Luminescent Cell Viability Assay Kit. Briefly, $100 \mu \mathrm{L}$ of CellTiter-Glo working reagent was added to each well and mixed on a microplate shaker for 10 mins. Luminescence was monitored using a luminescence microplate reader.

\section{Flow cytometric analysis of cell-derived microparticles}

Cell-derived microparticles were assessed by flow cytometric analysis (14). Briefly, EAhy926 cells were exposed to TNF $\pm \mathrm{CHX}$ for 24 hours after pretreatment with curcumin for one hour. Cell culture supernatant was collected and stained with Annexin-V-FITC and Annexin-V binding buffer. Samples were then mixed with a known concentration of $2.5 \mu \mathrm{m}$ AlignFlow $^{\mathrm{TM}}$ flow cytometry alignment beads (Invitrogen, Australia) as an internal control, and 50,000 events were analysed using the FACSCalibur flow cytometer (BD Biosciences, Australia) and WEASEL Software Version 3.0.2 (Walter and Eliza Hall Institute, Australia). Particulates $(<1 \mu \mathrm{m})$ were gated as a population using the upper limits of 0.8 $\mu \mathrm{m}$ and lower limits of $1.1 \mu \mathrm{m}$ using calibrated latex beads with particle size of 0.8 and $1.1 \mu \mathrm{m}$ (Sigma-Aldrich, Australia). The number of positive Annexin-V-FITC fluorescent events within the gated population was considered as microparticles. The number of positive events was normalised to the internal control beads and was expressed as fold change relative to control.

\section{Flow cytometric analyses for cell adhesion molecules expressions}

Surface expressions of cell adhesion molecule were performed using flow cytometry. Briefly, EAhy926 cells were exposed to TNF for 4 hours after pretreatment with curcumin for one hour. Cells were harvested and collected by centrifugation at $500 \times \mathrm{g}$ for $5 \mathrm{mins}$. The cell pellet was stained with CD54-PE in serum containing medium for 30 mins on ice. The pellet was then washed three times, and the samples were subjected to flow cytometric analyses. Ten thousand (10,000) cells were analysed using the FACSCalibur flow cytometer. The results are presented as mean fluorescence intensity and the percentage of cells with positive staining. 


\begin{abstract}
Monocyte-endothelial adhesion assay
Monocyte-endothelial adhesion assay was performed as previously described (21). Briefly, the confluent endothelial monolayer was stimulated with TNF for 4 hours after pretreatment with curcumin for one hour. The cells were then co-cultured with THP- 1 cells at $37^{\circ} \mathrm{C}$ for $30 \mathrm{~min}$. After the incubation period, the wells were washed gently three times with PBS and fixed with 3\% buffered paraformaldehyde for 20 minutes. The cells were then stained with $0.2 \%$ crystal violet in $20 \%$ methanol for 5 minutes. The excess crystal violet stain was rinsed with distilled water for 4-5 times and air-dried. Glacial acetic acid (33\%) in Milli-Q water was added to extract the crystal violet stain. The absorbance was then measured at $595 \mathrm{~nm}$.
\end{abstract}

\section{Flow cytometric analysis for Annexin V/PI staining}

Apoptosis was detected by flow cytometric analysis using a commercial FITC Annexin V apoptosis detection kit. Briefly, EAhy926 cells were exposed to TNF plus CHX for 24 hours after pretreatment with curcumin for one hour. Both detached and the attached monolayer cells were collected by centrifugation at $500 \mathrm{x} \mathrm{g}$ for 5 minutes and resuspended in Annexin- $\mathrm{V}$ binding buffer. The cells were then incubated with FITC-Annexin-V and propidium iodide (PI) for 15 minutes, and 10,000 cells were analysed within 1 hour using the FACSCalibur flow cytometer. The percentage of apoptotic cells were quantified by combining both early (FITC-Annexin- $\mathrm{V}^{+} / \mathrm{PI}^{-}$) and late (FITC-Annexin- $\mathrm{V}^{+} / \mathrm{PI}^{+}$) apoptotic cells, while negative staining for both FITC-Annexin-V and PI were recognised as healthy cells.

\section{Flow cytometric analysis of mitochondrial membrane potential}

Mitochondrial membrane potential was assessed by flow cytometric analysis (22). Briefly, EAhy926 cells were exposed to TNF plus CHX for 24 hours after pretreatment with curcumin for one hour. Both detached and the attached monolayer cells were collected by centrifugation at $500 \mathrm{x}$ g for 5 minutes. The cell pellet was then incubated in JC-1 solution for 15 minutes at $37^{\circ} \mathrm{C}$, and 10,000 cells were analysed using the FACSCalibur flow cytometer. Carbonyl cyanide m-chlorophenyl hydrazone (CCCP) $(500 \mu \mathrm{M})$ was used as a positive control for gating.

\section{STATISTICAL ANALYSES}

Statistical comparisons were performed using GraphPad Version 5.02 (USA). The data was analysed by one-way analysis of variance (ANOVA) followed by Newman-Keuls post hoc test. Data was expressed as mean \pm S.E.M. $\mathrm{P}<0.05$ was considered as statistically significant.

\section{RESULTS}

\section{TNF enhanced microparticle release}

The activities of TNF can be switched from cell activation to cell death in the presence of CHX. To examine the effects of TNF, EAhy926 cells were treated with TNF \pm CHX for 24 hours, and cell viability was evaluated using the MTT dye reduction assay. Neither TNF (1-10 $\mathrm{ng} / \mathrm{mL})$, nor CHX (100 ng/mL) showed significant influences on cell viability (Fig. 1A). As expected (8), treatment with TNF $(10 \mathrm{ng} / \mathrm{mL})$ plus CHX $(100 \mathrm{ng} / \mathrm{mL})$ significantly exacerbated cell death in EAhy926 cells $(\mathrm{P}<0.05)$.

Cell-derived microparticles can be released following cell activation and apoptosis (14). To study the effects of TNF on microparticle release, EAhy926 cells were treated with TNF and/or CHX for 24 hours, and the microparticle levels in cell culture supernatant were evaluated by flow cytometry. Microparticles are commonly distinguished by Annexin $\mathrm{V}$ positive (i.e. phosphatidylserine (PS) positive) submicron membrane vesicles $(<1 \mu \mathrm{m}$ in diameter) (14). Therefore, the microparticles in this study were defined as vesicles positive to FITC-Annexin V and their sizes were less than $1 \mu \mathrm{m}$ (Fig. 1B-C). Our results showed that TNF $(10 \mathrm{ng} / \mathrm{mL})$ increased the numbers of microparticles by $2.05 \pm 0.37$ fold compared to the control group (Fig. 1D). The combined treatment of TNF $(10 \mathrm{ng} / \mathrm{mL})$ and $\mathrm{CHX}$ $(100 \mathrm{ng} / \mathrm{mL})$ significantly elevated the microparticle level to $3.12 \pm 0.11$ fold (Fig. 1E). 


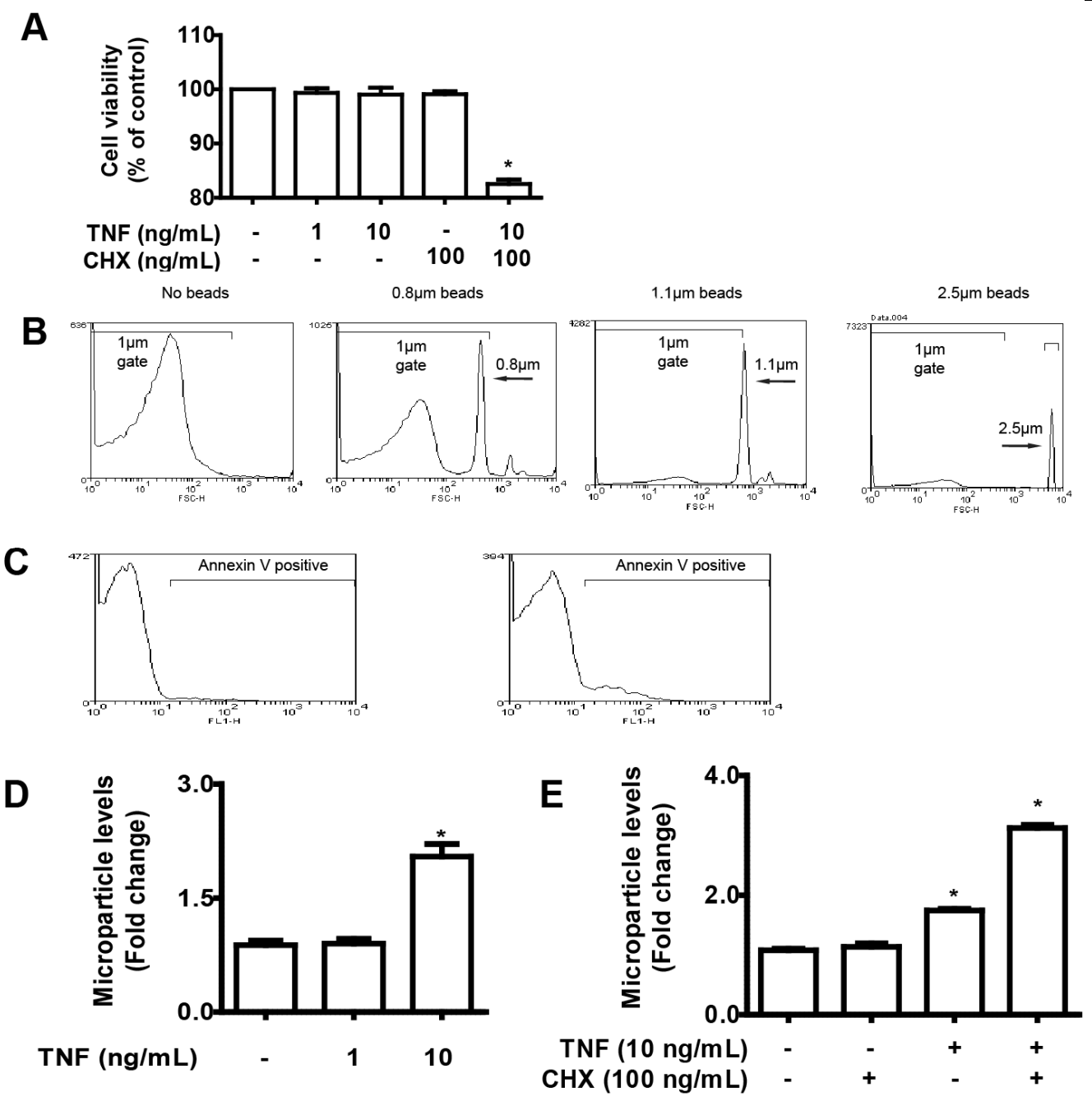

Figure 1. Effects of TNF and cycloheximide on endothelial cell viability and microparticle release. (A) Cell viability, as determined by MTT dye reduction assay, following incubation with TNF, CHX or TNF plus CHX for 24 hours in EAhy926 cells. Cell viability was expressed as a percentage compared to control. All results were expressed as mean \pm S.E.M. from three separate experiments. ${ }^{*} \mathrm{P}<0.05$ compared to control. For the detection of microparticles, (B) latex beads of different sizes $(0.8$ and $1.1 \mu \mathrm{m})$ were used to gate particles with diameter less than $1 \mu \mathrm{m}$ from the population, while $2.5 \mu \mathrm{m}$ beads were used as internal control beads. (C) Gated particles $(<1 \mu \mathrm{m})$ in the left panel were stained with FITC-Annexin V to generate a sub-population that is positively stained with Annexin V as shown in the right panel. Using this method, microparticle levels in the cell culture supernatant of EAhy926 endothelial cells treated with (D) TNF and (E) TNF plus CHX for 24 hours were analysed by flow cytometric analysis following staining with FITC-Annexin V. The number of Annexin V positive microparticles was normalised with the internal control beads and was expressed as fold change relative to control. All results were expressed as mean \pm S.E.M. from quadruplicate samples. * $\mathrm{P}<0.05$ compared to control.

\section{Curcumin reduced TNF-enhanced microparticle release}

Curcumin treatment up to $5 \mu \mathrm{M}$ for 24 hours showed $>95 \%$ cell viability (Fig. $2 \mathrm{~A}$ ). To evaluate the effects of curcumin on TNF $\pm \mathrm{CHX}$-enhanced microparticle release, EAhy926 cells were pretreated with curcumin $(1-5 \mu \mathrm{M})$ for one hour and then incubated with TNF $(10 \mathrm{ng} / \mathrm{mL}) \pm \mathrm{CHX}$
(100 ng/mL) for 24 hours. Our results showed that curcumin concentration-dependently reduced TNF-enhanced microparticle release (Fig. 2B). Similar results were also observed in the TNF plus CHX treatment group where curcurmin reduced the microparticle levels in a concentration-dependent manner (Fig. 2C). 
A

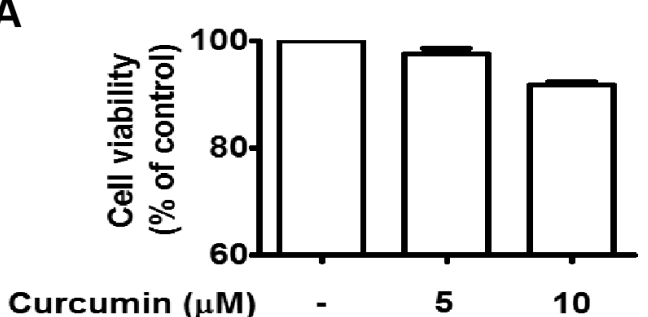

B
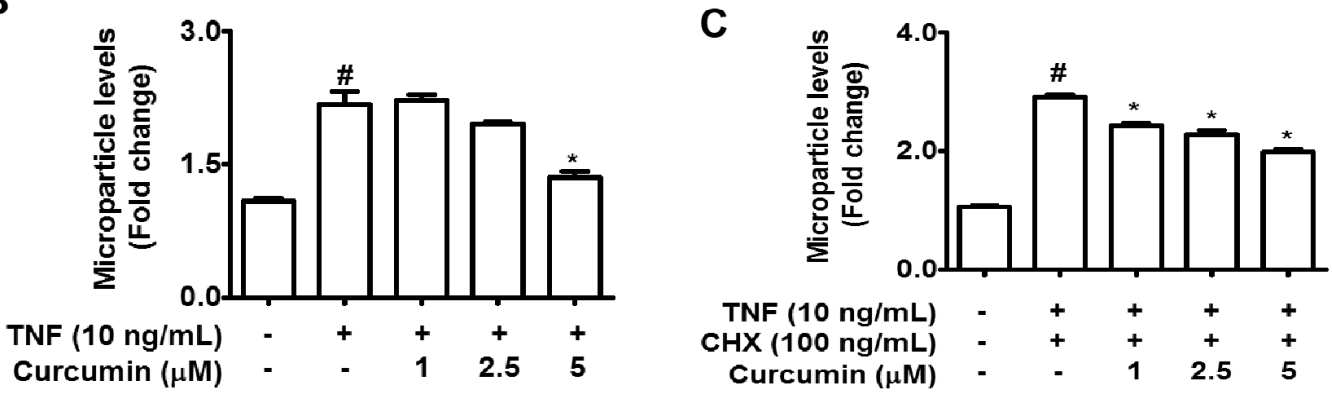

Figure 2. Effects of curcumin on EAhy926 cell microparticle levels. (A) Cell viability, as determined by MTT dye reduction assay, following incubation with curcumin for 24 hours in EAhy926 cells. Cell viability was expressed as a percentage compared to control. All results were expressed as mean \pm S.E.M. from three separate experiments. To determine the effects of curcumin on microparticle levels, EAhy926 endothelial cells were pretreated with curcumin for one hour and then exposed to (B) TNF alone and (C) TNF plus CHX for 24 hours. Cell culture supernatant was analysed by flow cytometric analysis following staining with FITC-Annexin V. The number of Annexin V positive microparticles was normalised with the internal control beads and was expressed as fold change relative to control. All results were expressed as mean \pm S.E.M. from quadruplicate samples. \# $\mathrm{P}<0.05$ compared to control. ${ }^{*} \mathrm{P}<0.05$ compared to TNF alone or TNF plus CHX treated-group.

\section{Curcumin attenuated endothelial cell activation}

Endothelial cell activation refers to a change in the endothelial cell phenotype in response to cellular stress and is denoted by an increase in endothelial-leukocyte interactions and the up-regulation of cell adhesion molecules (e.g. ICAM-1, VCAM-1 and E-selectin) (23). To investigate the effects of curcumin on endothelial cell activation, ICAM-1 surface expression and monocyte adhesion to endothelial monolayer were evaluated. EAhy926 cells were pretreated with curcumin for 1 hour and then exposed to TNF (10 $\mathrm{ng} / \mathrm{mL}$ ) for 4 hours. As shown in Fig 3A-B, pretreatment with curcumin $(2.5 \quad-5 \mu M)$ significantly attenuated the surface expression of ICAM-1 and reduced the proportion of cells with up-regulated ICAM-1 expression. To demonstrate the adhesion capacity of TNF-stimulated EAhy 926 cells, THP-1 monocytic cells were co-cultured with EAhy926 cells. Our results showed that curcumin $(2.5-5 \mu \mathrm{M})$ significantly reduced the adhesion of
THP-1 monocytic cells onto the TNF-stimulated EAhy926 endothelial monolayer (Fig. 3C).

\section{Curcumin attenuated TNF plus CHX-induced cell death}

As described, CHX sensitised TNF-induced cytotoxicity and exacerbated microparticle release from EAhy926 cells, while pretreatment with curcumin reduced TNF plus CHX-induced microparticle release. To study the effects of curcumin on TNF plus CHX-induced endothelial cell death, EAhy926 cells were pretreated with curcumin for 1 hour and exposed to TNF (10 $\mathrm{ng} / \mathrm{mL}$ ) plus CHX (100 ng/mL) for 24 hours. As revealed by the MTT dye reduction assay, curcumin $(2.5-5 \mu \mathrm{M})$ significantly attenuated the cytotoxic effects of TNF plus CHX in EAhy926 cells in a concentration-dependent manner $(\mathrm{P}<0.05)$ (Fig. 4A). Comparable results were observed using a commercial cell viability kit measuring ATP content $(\mathrm{P}<0.05)$ (Fig. 4B). 

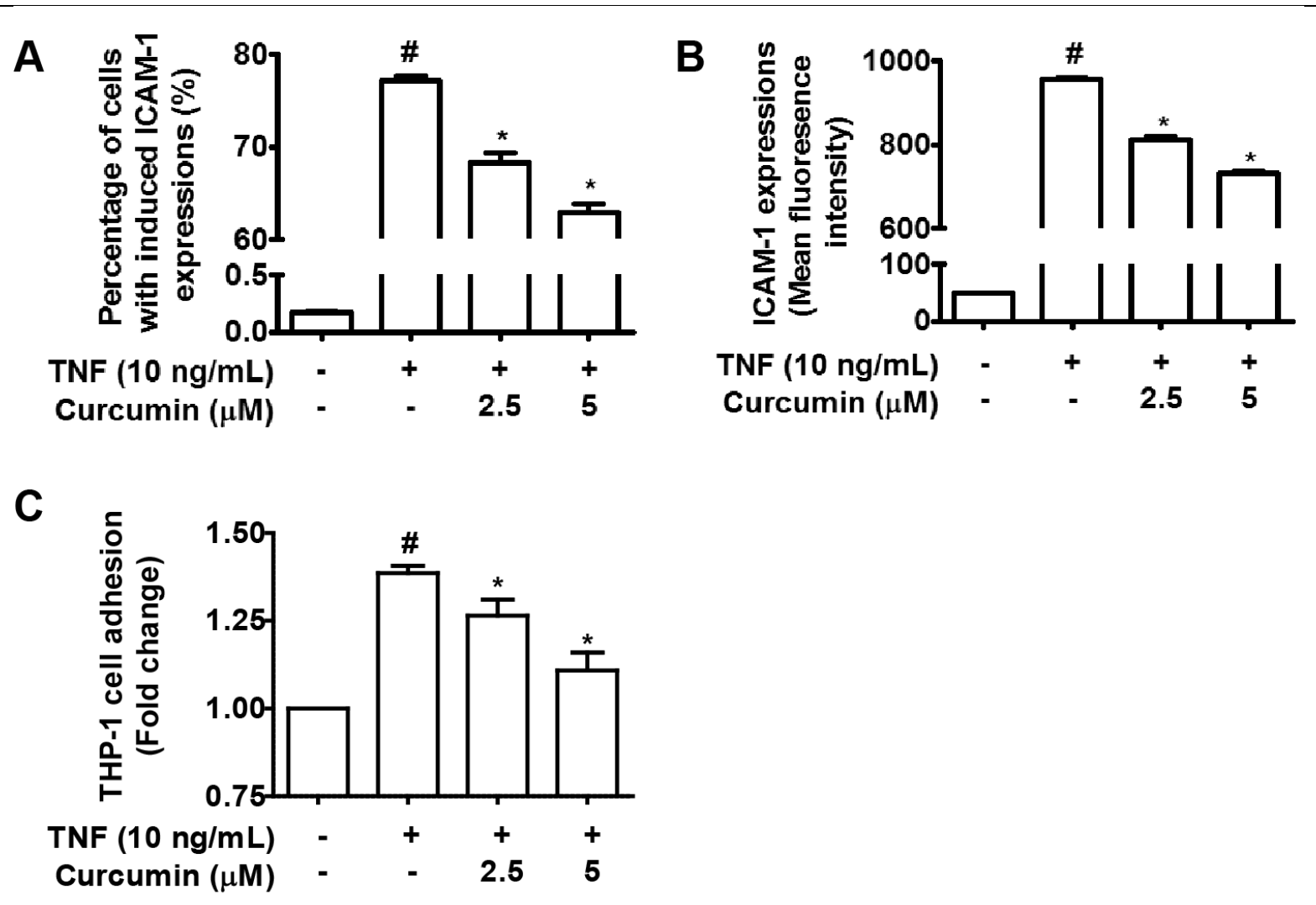

Figure 3. Curcumin attenuated endothelial cell activation. To determine the effects of curcumin on ICAM-1 expressions, EAhy926 cells were pretreated with vehicle or curcumin (final DMSO concentration: $0.1 \%$ ) for 1 hour and then exposed to TNF for 4 hours. Cells were analysed by flow cytometric analysis following staining with PE-CD54 (ICAM-1). The results were expressed as (A) a percentage of induced ICAM-1 cells and (B) mean fluorescence intensity in arbitrary units. All results were expressed as mean \pm S.E.M. from quadruplicate samples. \# P $<0.05$ compared to blank. * $\mathrm{P}<0.05$ compared to TNF treated-group. (C) The adhesion of THP-1 cells to EAhy 926 cells was assessed by crystal violet staining. Cell adhesion was expressed as fold change compared to control. All results were expressed as mean \pm S.E.M. from three separate experiments. $\# \mathrm{P}<0.05$ compared to control. * $\mathrm{P}<0.05$ compared to TNF treated-group.

\section{Curcumin attenuated TNF plus CHX-induced apoptotic events}

It is known that CHX can exacerbate TNF-induced apoptosis (24). To study the influence of curcumin on TNF plus CHX-mediated apoptotic events, EAhy926 cells were pretreated with curcumin for 1 hour and then exposed to the combination of TNF $(10 \mathrm{ng} / \mathrm{mL})$ and CHX (100 ng/mL) for 24 hours. Our results demonstrated that the exposure of the cells to TNF plus CHX significantly elevated the proportion of EAhy926 cells with positive Annexin-V staining from $1.99 \pm 0.05 \%$ to $15.82 \pm$ $0.40 \%$. Pretreatment with curcumin $(2.5-5 \mu \mathrm{M})$ significantly attenuated the apoptotic effects in a concentration-dependent manner $(\mathrm{P}<0.05)$ (Fig $4 C)$.
Apoptosis can also be described by the disruption of the mitochondrial membrane potential $\left(\Delta \psi_{\mathrm{m}}\right)$. Using a fluorescence probe (i.e. JC-1) to measure mitochondrial potentials, our results revealed that the proportion of cells with depolarised $\Delta \psi_{\mathrm{m}}$ (as indicated by a reduction in JC-1 aggregate red to JC-1 monomer green fluorescence) were significantly increased in TNF plus CHX -treated EAhy926 cells from $21.11 \pm$ $1.39 \%$ to $37.97 \pm 1.73 \%$. Pretreatment with curcumin $(2.5-5 \mu \mathrm{M})$ concentration-dependently reduced the proportion of cells with depolarised $\Delta \psi_{\mathrm{m}}(\mathrm{P}<0.05)($ Fig $4 \mathrm{D})$. 

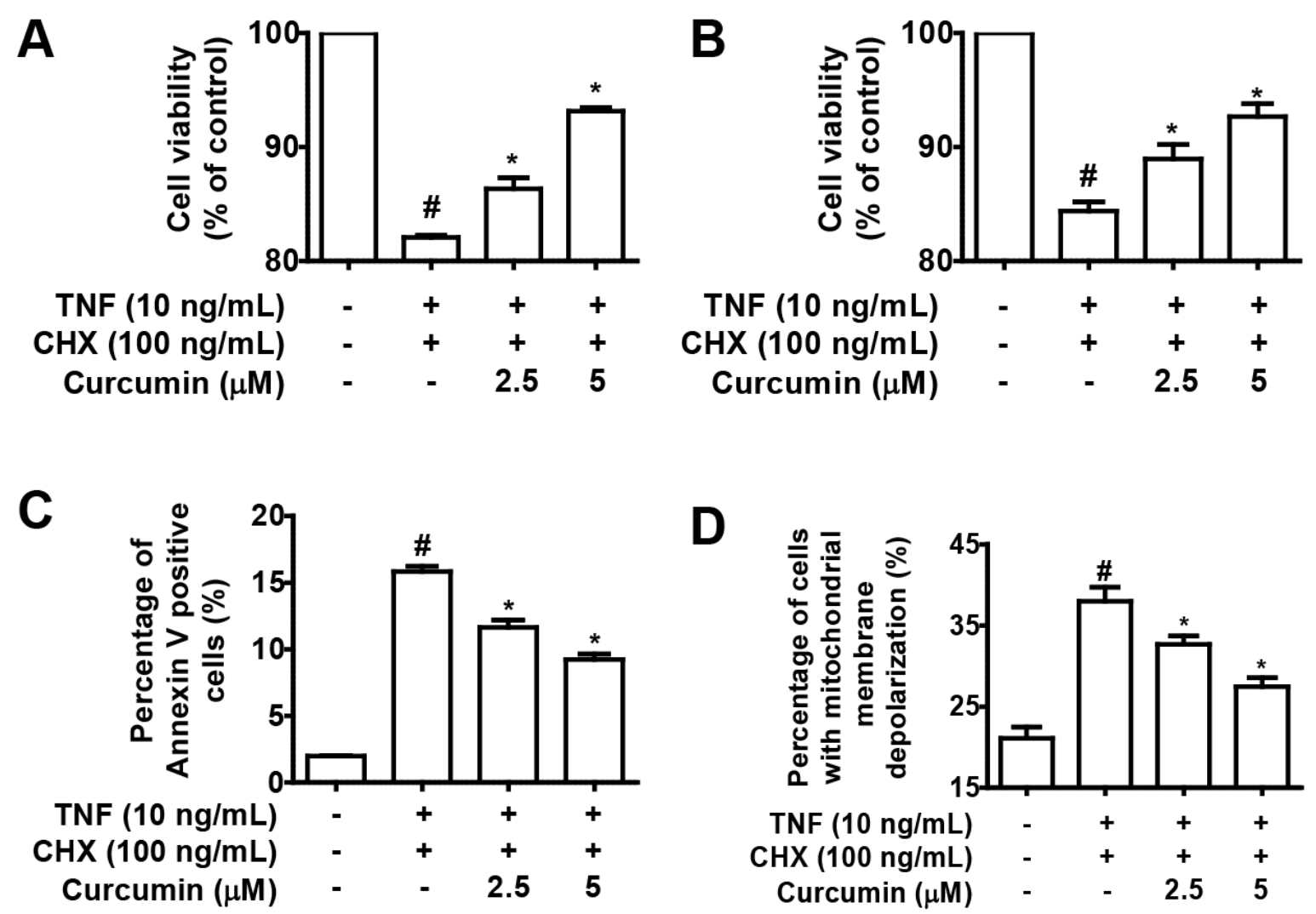

Figure 4. Curcumin attenuated endothelial cell death and apoptosis. Cell viability, as determined by (A) MTT dye reduction assay and (B) CellTiter ${ }^{\circledR}$-Glo cell viability assay, following incubation with TNF plus CHX for 20 hours in EAhy926 endothelial cells which were pretreated with vehicle or curcumin (final DMSO concentration: $0.1 \%$ ) for 1 hour. Cell viability was expressed as a percentage compared to control. All results were expressed as mean \pm S.E.M. from three separate experiments. \# $\mathrm{P}<0.05$ compared to control in the respective cell lines. $* \mathrm{P}<0.05$ compared to TNF plus CHX treated-group. To determine the effects of curcumin on endothelial cell apoptosis, (C) flow cytometric analysis with FITC-Annexin-V and PI staining revealed that curcumin attenuated alterations in the exposure of membrane phospholipid phosphatidylserine, while (D) JC-1 staining showed that curcumin reduced the proportion of cells with depolarised $\Delta \psi_{\mathrm{m}}$ (indicated by a reduction in JC-1 aggregate red to JC-1 monomer green fluorescence). All results were expressed as mean \pm S.E.M. from quadruplicate samples. \# $\mathrm{P}<0.05$ compared to control. $* \mathrm{P}<0.05$ compared to TNF plus CHX treated-group.

\section{DISCUSSION}

The elevated levels of circulating endothelial microparticles are associated with endothelial dysfunction observed in hypertension, coronary artery diseases, ischaemic stroke, diabetes and other cardiovascular events $(19,25-27)$. It has been suggested that these microparticles are implicated in the regulation of coagulation, oxidative stress, inflammation, angiogenesis and apoptosis $(18,28)$. Therefore, therapeutic agents targeting microparticle release is an emerging strategy for cardiovascular diseases. Natural products such as turmeric represent a promising resource for the discovery of agents against microparticle generation. Here, we present the first evidence regarding the inhibitory effects of curcumin, a major curcuminoid in turmeric, on microparticle release from endothelial cells undergoing cell activation and cell death. In this study, microparticles are identified by an apoptotic marker, Annexin V, together with size cutoff at $<1 \mu \mathrm{m}$, to minimize the interference of apoptotic bodies which are normally 1-4 $\mu \mathrm{m}$ in size (14).

Under physiological conditions, cells release basal levels of microparticles (18). However, excessive microparticle generation can be triggered upon cell activation and apoptosis $(18,29)$. In our 
study, TNF elevated microparticle formation and this was shown by the shedding of vesicles with submicron size carrying phosphatidylserine. Our results showed that the pretreatment of curcumin reduced microparticle formation from endothelial cells enhanced by TNF. TNF is a well-known inducer of cell activation (30). Similar to previous reports, we demonstrated that TNF- induced cell activation reflected by the increased expressions of ICAM-1 and adhesion of monocytes to the endothelial monolayer (7). Our results further showed that these features were attenuated by the pretreatment of curcumin. These results support previous findings that curcumin suppressed ICAM-1 expressions and the adhesion of monocytes to TNF-activated human umbilical vein endothelial cells (21,31-33). Current literature has reported that these inhibitory effects are related to the role of curcumin in modulating inflammation-associated pathways (e.g. NF- $\mathrm{BB}$ and MAPK pathways) (31,33-35). These inflammatory pathways are known to be actively involved in the generation of microparticles $(10,12)$. Further studies are warranted to evaluate the modulatory effects of curcumin on inflammatory pathways influencing microparticle generation.

We also showed that the release of microparticles by TNF was further exacerbated through co-incubation with $\mathrm{CHX}$, and this was attenuated by the pretreatment of curcumin. Cell death and apoptosis are important mechanisms involved in microparticle release. Similar to previous reports, we demonstrated that $\mathrm{CHX}$ exacerbated TNF-induced cytotoxicity (8). Our results further showed that curcumin exerted cytoprotective and anti-apoptotic effects in endothelial cells treated with TNF plus CHX. This is similar to previous reports showing that curcumin protects endothelial, neuronal and retinal epithelial cells from damage caused by oxidative stress and various toxins (36-38). We speculate that the reduction in microparticle levels under cell death conditions may be associated with the anti-apoptotic activities of curcumin. Further mechanistic studies are needed to address the action of curcumin on microparticle release which can be related to microparticle formation, or vesicle transport and release.

In conclusion, curcumin reduced microparticle release from endothelial cells, which can be associated to its inhibitory effects against cell activation and cell death. These findings support curcumin as a promising nutraceutical candidate for vascular inflammatory diseases.

\section{CONFLICT OF INTEREST}

The authors declare that they have no conflicts of interests.

\section{REFERENCES}

1. Aggarwal BB, Harikumar KB. Potential therapeutic effects of curcumin, the anti-inflammatory agent, against neurodegenerative, cardiovascular, pulmonary, metabolic, autoimmune and neoplastic diseases. Int J Biochem Cell Biol 2009; 41: 40-59

2. Omar EA, Kam A, Alqahtani A, Li KM, Razmovski-Naumovski V, Nammi S, Chan K, Roufogalis BD, Li GQ. Herbal medicines and nutraceuticals for diabetic vascular complications: mechanisms of action and bioactive phytochemicals. Curr Pharm Des 2010; 16: 3776-3807

3. Kam A, Li KM, Razmovski-Naumovski V, Nammi S, Chan K, Li Y, Li GQ. The protective effects of natural products on blood-brain barrier breakdown. Curr Med Chem 2012; 19: 1830-1845

4. Miriyala S, Panchatcharam M, Rengarajulu P. Cardioprotective effects of curcumin. Adv Exp Med Biol 2007; 595: 359-377

5. Aggarwal BB, Gupta SC, Sung B. Curcumin: an orally bioavailable blocker of TNF and other pro-inflammatory biomarkers. Br J Pharmacol 2013; 169: 1672-1692

6. McKellar GE, McCarey DW, Sattar N, McInnes IB. Role for TNF in atherosclerosis? Lessons from autoimmune disease. Nat Rev Cardiol 2009; 6: $410-417$

7. Burke-Gaffney A, Hellewell PG. Tumour necrosis factor-alpha-induced ICAM-1 expression in human vascular endothelial and lung epithelial cells: modulation by tyrosine kinase inhibitors. $\mathrm{Br} \mathrm{J}$ Pharmacol 1996; 119: 1149-1158

8. Nolop KB, Ryan US. Enhancement of tumor necrosis factor-induced endothelial cell injury by cycloheximide. Am J Physiol 1990; 259: L123-129

9. Aoki M, Kuwamura M, Kotani T, Katamoto H, Kubo K, Nomura K, Sasaki N, Ohashi F. In vitro cytotoxicity of recombinant human-TNF-alpha and actinomycin D on canine normal and tumor cells. J Vet Med Sci 1998; 60: 1087-1091

10. Johnson BL, 3rd, Goetzman HS, Prakash PS, Caldwell CC. Mechanisms underlying mouse TNF-alpha stimulated neutrophil derived microparticle generation. Biochem Biophys Res Commun 2013; 437: 591-596

11. Jimenez JJ, Jy W, Mauro LM, Soderland C, Horstman LL, Ahn YS. Endothelial cells release phenotypically and quantitatively distinct microparticles in activation and apoptosis. Thromb 
Res 2003; 109: 175-180

12. Curtis AM, Wilkinson PF, Gui M, Gales TL, Hu E, Edelberg JM. p38 mitogen-activated protein kinase targets the production of proinflammatory endothelial microparticles. J Thromb Haemost 2009; 7: 701-709

13. Combes V, Simon AC, Grau GE, Arnoux D, Camoin L, Sabatier F, Mutin M, Sanmarco M, Sampol J, Dignat-George F. In vitro generation of endothelial microparticles and possible prothrombotic activity in patients with lupus anticoagulant. J Clin Invest 1999; 104: 93-102

14. van der Heyde HC, Gramaglia I, Combes V, George TC, Grau GE. Flow cytometric analysis of microparticles. Methods Mol Biol 2011; 699: 337-354

15. Andriantsitohaina R, Gaceb A, Vergori L, Martinez MC. Microparticles as regulators of cardiovascular inflammation. Trends Cardiovasc Med 2012; 22: 88-92

16. Morel O, Jesel L, Freyssinet JM, Toti F. Cellular mechanisms underlying the formation of circulating microparticles. Arterioscler Thromb Vasc Biol 2011; 31: $15-26$

17. Tushuizen ME, Diamant M, Sturk A, Nieuwland R. Cell-derived microparticles in the pathogenesis of cardiovascular disease: friend or foe? Arterioscler Thromb Vasc Biol 2011; 31: 4-9

18. Lovren F, Verma S. Evolving role of microparticles in the pathophysiology of endothelial dysfunction. Clin Chem 2013; 59: 1166-1174

19. Viera AJ, Mooberry M, Key NS. Microparticles in cardiovascular disease pathophysiology and outcomes. J Am Soc Hypertens 2012; 6: 243-252

20. Kam A, Li KM, Razmovski-Naumovski V, Nammi $\mathrm{S}$, Chan K, Li GQ. Combination of TNF-alpha, homocysteine and adenosine exacerbated cytotoxicity in human cardiovascular and cerebrovascular endothelial cells. Cell Physiol Biochem 2012; 30: 805-814

21. Coban D, Milenkovic D, Chanet A, Khallou-Laschet J, Sabbe L, Palagani A, Vanden Berghe W, Mazur A, Morand C. Dietary curcumin inhibits atherosclerosis by affecting the expression of genes involved in leukocyte adhesion and transendothelial migration. Mol Nutr Food Res 2012; 56: 1270-1281

22. Kam A, Li KM, Razmovski-Naumovski V, Nammi S, Chan K, Li GQ. Gallic acid protects against endothelial injury by restoring the depletion of DNA methyltransferase 1 and inhibiting proteasome activities. Int J Cardiol 2014; 171: 231-242

23. Sima AV, Stancu CS, Simionescu M. Vascular endothelium in atherosclerosis. Cell Tissue Res 2009; 335: 191-203

24. Polunovsky VA, Wendt $\mathrm{CH}$, Ingbar DH, Peterson MS, Bitterman PB. Induction of endothelial cell apoptosis by TNF alpha: modulation by inhibitors of protein synthesis. Exp Cell Res 1994; 214: 584-594

25. Lee ST, Chu K, Jung KH, Kim JM, Moon HJ, Bahn JJ, Im WS, Sunwoo J, Moon J, Kim M, Lee SK, Roh JK. Circulating CD62E+ microparticles and cardiovascular outcomes. PLoS One 2012; 7: e 35713

26. Werner N, Wassmann S, Ahlers P, Kosiol S, Nickenig G. Circulating CD31+/annexin V+ apoptotic microparticles correlate with coronary endothelial function in patients with coronary artery disease. Arterioscler Thromb Vasc Biol 2006; 26: 112-116

27. Bulut D, Maier K, Bulut-Streich N, Borgel J, Hanefeld C, Mugge A. Circulating endothelial microparticles correlate inversely with endothelial function in patients with ischemic left ventricular dysfunction. J Card Fail 2008; 14: 336-340

28. Burger D, Schock S, Thompson CS, Montezano AC, Hakim AM, Touyz RM. Microparticles: biomarkers and beyond. Clin Sci (Lond) 2013; 124: 423-441

29. Bebawy M, Roseblade A, Luk F, Rawling T, Ung A, Grau GE. Cell-derived microparticles: new targets in the therapeutic management of disease. J Pharm Pharm Sci 2013; 16: 238-253

30. Cabal-Hierro L, Lazo PS. Signal transduction by tumor necrosis factor receptors. Cell Signal 2012; 24: $1297-1305$

31. Kumar A, Dhawan S, Hardegen NJ, Aggarwal BB. Curcumin (Diferuloylmethane) inhibition of tumor necrosis factor (TNF)-mediated adhesion of monocytes to endothelial cells by suppression of cell surface expression of adhesion molecules and of nuclear factor-kappaB activation. Biochem Pharmacol 1998; 55: 775-783

32. Gupta B, Ghosh B. Curcuma longa inhibits TNF-alpha induced expression of adhesion molecules on human umbilical vein endothelial cells. Int J Immunopharmacol 1999; 21: 745-757

33. Kim YS, Ahn Y, Hong MH, Joo SY, Kim KH, Sohn IS, Park HW, Hong YJ, Kim JH, Kim W, Jeong MH, Cho JG, Park JC, Kang JC. Curcumin attenuates inflammatory responses of TNF-alpha-stimulated human endothelial cells. J Cardiovasc Pharmacol 2007; 50: 41-49

34. Wang J, Dong S. ICAM-1 and IL-8 are expressed by DEHP and suppressed by curcumin through ERK and p38 MAPK in human umbilical vein endothelial cells. Inflammation 2012; 35: 859-870

35. Yen FL, Tsai MH, Yang CM, Liang CJ, Lin CC, Chiang YC, Lee HC, Ko HH, Lee CW. Curcumin nanoparticles ameliorate ICAM-1 expression in TNF-alpha-treated lung epithelial cells through p47 (phox) and MAPKs/AP-1 pathways. PLoS One 2013; 8: e63845

36. Woo JM, Shin DY, Lee SJ, Joe Y, Zheng M, Yim JH, Callaway Z, Chung HT. Curcumin protects retinal pigment epithelial cells against oxidative stress via 
induction of heme oxygenase-1 expression and reduction of reactive oxygen. Mol Vis 2012; 18: 901-908

37. Chen J, Tang XQ, Zhi JL, Cui Y, Yu HM, Tang EH, Sun SN, Feng JQ, Chen PX. Curcumin protects PC12 cells against 1-methyl-4-phenylpyridinium ion-induced apoptosis
bcl-2-mitochondria-ROS-iNOS pathway. Apoptosis 2006; 11: 943-953

38. Han J, Pan XY, Xu Y, Xiao Y, An Y, Tie L, Pan Y, Li XJ. Curcumin induces autophagy to protect vascular endothelial cell survival from oxidative stress damage. Autophagy 2012; 8: 812-825 